\title{
Two-dimensional performance of MIPAS observation modes in the upper-troposphere/lower-stratosphere
}

\author{
M. Carlotti ${ }^{1}$, E. Castelli ${ }^{2,3}$, and E. Papandrea ${ }^{1}$ \\ ${ }^{1}$ Dipartimento di Chimica Fisica e Inorganica, University of Bologna, Viale Risorgimento 4, 40136 Bologna, Italy \\ ${ }^{2}$ Istituto di Scienze dell' Atmosfera e del Clima, CNR, Via P. Gobetti 101, 40129 Bologna, Italy \\ ${ }^{3}$ Istituto per le Applicazioni del Calcolo "Mauro Picone”, CNR, Via Madonna del Piano 10, \\ 50019 Sesto Fiorentino (FI), Italy \\ Received: 25 May 2010 - Published in Atmos. Meas. Tech. Discuss.: 8 July 2010 \\ Revised: 14 February 2011 - Accepted: 22 February 2011 - Published: 25 February 2011
}

\begin{abstract}
In this paper we analyze the performance of the three MIPAS (Michelson Interferometer for Passive Atmospheric Sounding) observation modes that sound the UpperTroposphere/Lower-Stratosphere (UT/LS) region. The twodimensional (2-D) tomographic retrieval approach is assumed to derive the atmospheric field of geophysical parameters. For each observation mode we have calculated the 2-D distribution of the information load quantifier relative to the main MIPAS targets. The performance of the observation modes has been evaluated in terms of strength and spatial coverage of the information-load distribution along the full orbit. The indications of the information-load analysis has been validated with simulated retrievals based on the observational parameters of real orbits. In the simulation studies we have assessed the precision and the spatial (both horizontal and vertical) resolution of the retrieval products. The performance of the three observation modes has been compared for the MIPAS main products in both the UT/LS and the extended altitude range. This study shows that the two observation modes that were specifically designed for the UT/LS region are actually competitive with the third one, designed for the whole stratosphere, up to altitudes that far exceed the UT/LS. In the UT/LS the performance of the two specific observation modes is comparable even if the best performance in terms of horizontal resolution is provided by the observation mode that was excluded by the European Space Agency (ESA) from the current MIPAS duty cycle. This paper reports the first application of the information-load analysis and highlights the worthiness of this approach to make qualitative considerations about retrieval potential and selection of retrieval grid.
\end{abstract}

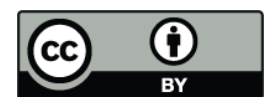

Correspondence to: M. Carlotti (carlotti@fci.unibo.it)

\section{Introduction}

The Michelson Interferometer for Passive Atmospheric Sounding (MIPAS) in its present configuration can measure the atmosphere with seven different observation modes. Two of them have been expressly designed to sound the UpperTroposphere/Lower-Stratosphere (UT/LS) region although one of them has been operated for only 15 test orbits and then excluded from the operational duty cycle. The MIPAS "nominal" observation mode also sounds the UT/LS region with a vertical sampling which is similar to the one of the two specific modes. The three observation modes mainly differ in the limb scanning pattern at upper altitudes. Therefore, due to the different measuring time required by a single limb scan, they also operate a different sampling of the atmosphere in the horizontal domain.

When the MIPAS observation modes were designed, consolidated tools were not yet available to evaluate the consistency between geometrical limb steps and spatial resolution of the retrieval products. The two-dimensional (2-D) retrieval strategy has introduced the possibility to set the horizontal separation among the altitude profiles of the target quantity. The horizontal resolution then becomes a property that qualifies the retrieval products in addition to their vertical resolution and precision. In this paper we analyze the performance of the three observation modes that sound the UT/LS when a 2-D tomographic approach (Carlotti et al., 2001) is exploited to derive the field of atmospheric parameters. The relative merit of each observation mode will be evaluated in terms of retrieval precision and spatial resolution of the main MIPAS retrieval products.

The features of the MIPAS experiment that are relevant for our study are reported in Sect. 2 while Sect. 3 provides a survey of the mathematical tools exploited in the following sections. In the first stage of our analysis (described in

Published by Copernicus Publications on behalf of the European Geosciences Union. 
Sect. 4) we have used the recently introduced informationload $(\Omega)$ analysis (Carlotti and Magnani, 2009) to calculate the 2-D distribution of the $\Omega$ quantifier for the main MIPAS geophysical targets. The $\Omega$ distribution maps assess the actual target-dependent atmospheric sampling of the measurements and permit to compare the performance of the observation modes in terms of strength and spatial coverage of the $\Omega$ distribution along the full orbit. In a second stage (described in Sect. 5) we have carried out simulated 2-D retrievals based on the observational parameters of real orbits measured with the three observation modes. The analysis carried out on simulated observations permits to evaluate the achieved precision by comparing the retrieved values of the parameters with the reference values used to generate the simulated observations. The algorithm also provides averaging kernels (Rodgers, 2000) that are used to evaluate both the horizontal and the vertical resolution of the retrieval products (Carlotti et al., 2007). The overall performance of the considered observation modes is then compared in terms of $\Omega$ distribution, precision, and spatial resolution of the retrieval products in both the full altitude range and in the UT/LS region. Finally, a summary of the main findings of this study and concluding remarks are reported in Sect. 6.

\section{The MIPAS experiment}

MIPAS has been developed by the European Space Agency (ESA) for the study of atmospheric composition. MIPAS measures the atmospheric emission from a nearly polar orbit onboard the ENVISAT satellite. The atmospheric emission, in the spectral interval from $680 \mathrm{~cm}^{-1}$ to $2410 \mathrm{~cm}^{-1}$, is observed by MIPAS with the limb-scanning observation technique. In its original configuration the maximum optical path difference of the interferometer was $20 \mathrm{~cm}$ that corresponds to an unapodized spectral resolution of $0.025 \mathrm{~cm}^{-1}$ Fourier Transform, and $0.035 \mathrm{~cm}^{-1}$ Full Width at Half Maximum (FWHM). Starting from January 2005 a "new" configuration has been implemented in which the instrument is operated at $41 \%$ of its maximum spectral resolution. The study reported in this paper refers to this configuration that is fully operational since January 2008.

In its present configuration MIPAS can measure the atmosphere with seven different observation modes. Two of them (denoted as UTLS-1 and UTLS-2) have been expressly designed to sound the UT/LS region. The MIPAS "nominal" observation mode (denoted as NOM and operated for about $80 \%$ of the instrument's measuring time) extends to the whole stratosphere but also sounds the UT/LS region. All the three observation modes have identical spectral resolution and quality of the signals; they measure consecutive backward-looking limb-scans with the line of sight approximately lying in the orbit plane. They differ in both vertical steps and number of observation geometries. And therefore, due to the different measuring time required by a single limb scan, they also operate different samplings of the atmosphere in the horizontal domain. The limb-scanning pattern of NOM consists of 27 observation geometries. Starting from the bottom the first 11 tangent altitudes of NOM are separated by $1.5 \mathrm{~km}$; altitude steps of 2,3 , and $4 \mathrm{~km}$ space out the next groups of 5, 5, and 6 tangent altitudes respectively. This limb pattern, combined with the ENVISAT orbit period of $101 \mathrm{~min}$, generates about 96 limb-scans per orbit with an average separation of about $415 \mathrm{~km}(\sim 3.7 \mathrm{deg})$ between consecutive limb-scans. For UTLS-1 the limb-scanning pattern consists of 19 observation geometries; the first 9 tangent altitudes are separated by $1.5 \mathrm{~km} ; 2,3,4$, and $4.5 \mathrm{~km}$ space out the next groups of 3,2, and 4 tangent altitudes respectively. This limb pattern generates about 125 limb-scans per orbit separated by about $320 \mathrm{~km}(\sim 2.9 \mathrm{deg})$. For NOM and UTLS1 the limb-scanning patterns are shifted in altitude along the orbit following a model that imitates the varying altitude of the tropopause as a function of latitude. Finally, in UTLS2 the limb-scanning pattern consists of 11 observation geometries; tangent altitudes are separated by $2 \mathrm{~km}$ from 12 to $20 \mathrm{~km}, 3$ and $4 \mathrm{~km}$ space out the next two groups of three tangent altitudes up to a maximum of $42 \mathrm{~km}$. This pattern generates about 213 limb-scans per orbit separated by about $190 \mathrm{~km}(\sim 1.7 \mathrm{deg})$. The UTLS-2 has been operated for a very limited number of orbits (15). The three panels of Fig. 1 show the layout of MIPAS tangent points along orbits measured with NOM (top), UTLS-1 (middle) and UTLS-2 (bottom) respectively. In Fig. 1 the altitude of tangent points is plotted as a function of the Orbital Coordinate (OC) defined as the polar angle originating at the most northern point of the orbit and spanning the orbit plane over its 360 deg extension. We can appreciate in Fig. 1 few minor gaps that occur in the horizontal sampling of the atmosphere of the three observation modes. Besides, the UTLS-1 shows a major gap that is present (even if at different positions) in all the orbits recorded with this observation mode. Relevant features of MIPAS are summarized in Table 1; a detailed description of the experiment can be found in Fischer et al. (2008).

\section{Mathematical tools}

\subsection{Retrieval strategy}

MIPAS spectra are analyzed by the ESA ground processor that determines, at the tangent points of each limb scan, the values of pressure, temperature and Volume Mixing Ratio (VMR) of six key atmospheric species $\left(\mathrm{H}_{2} \mathrm{O}, \mathrm{O}_{3}, \mathrm{HNO}_{3}\right.$, $\mathrm{CH}_{4}, \mathrm{~N}_{2} \mathrm{O}$ and $\mathrm{NO}_{2}$ ). For this purpose ESA employs a 1-D retrieval system (Ridolfi et al., 2000) that implements the global-fit algorithm (Carlotti, 1988). A 2-D algorithm, named Geo-fit (Carlotti et al., 2001), was subsequently developed for the analysis of MIPAS measurements and implemented in the Geofit MultiTarget Retrieval (GMTR) operational code (Carlotti et al., 2006); the study reported in this 


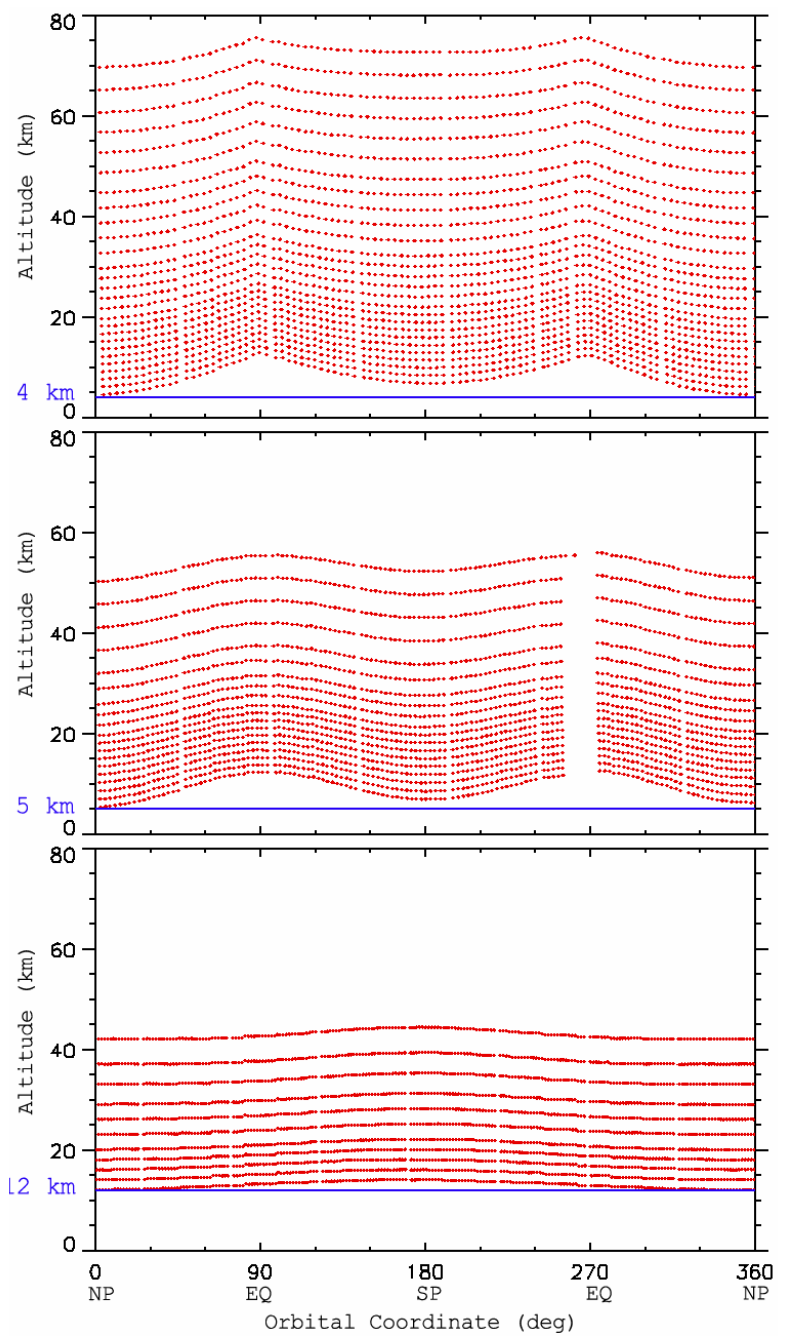

Fig. 1. Tangent points along orbits measured with NOM (top), UTLS-1 (middle) and UTLS-2 (bottom). Earth poles (NP, SP) and Equator (EQ) are marked on the abscissa axes.

paper refers to this kind of retrieval. The Geo-fit approach is based on the simultaneous inversion of observations selected from all the limb scans measured along a whole orbit. This strategy permits to merge the information on each retrieval parameter from different observation geometries and makes it possible to model the horizontal variability of the atmosphere. In the Geo-fit approach, the 2-D retrieval grid is fully independent from the measurement grid (i.e. the grid identified by the tangent points of the measurements). By exploiting this feature atmospheric profiles can be retrieved with horizontal separations that are different from those of the measured limb scans. Nevertheless, adopting the assumption that the information is mostly concentrated around the tangent point of the observations, the straightforward choice is a horizontal retrieval grid defined by the average geographical coordinates of the tangent points of the limb-scans. We will denote this choice as "natural" grid.
Table 1. Relevant features of the analyzed observation modes.

\begin{tabular}{|c|c|c|c|}
\hline & NOM & UTLS-1 & UTLS-2 \\
\hline Spectral resolution $\left(\mathrm{cm}^{-1}\right)$ & 0.0625 & 0.0625 & 0.0625 \\
\hline $\begin{array}{l}\text { Vertical/horizontal field } \\
\text { of view }^{1}(\mathrm{~km})\end{array}$ & $3 / 30$ & $3 / 30$ & $3 / 30$ \\
\hline $\begin{array}{l}\text { Variable altitude of } \\
\text { tangent points }\end{array}$ & Yes & Yes & No \\
\hline $\begin{array}{l}\text { No. of observation } \\
\text { geometries per limb-scan }\end{array}$ & 27 & 19 & 11 \\
\hline No. of limb-scans per orbit & 96 & 125 & 213 \\
\hline $\begin{array}{l}\text { Lowest/highest tangent } \\
\text { altitude }(\mathrm{km})\end{array}$ & $5 / 77$ & $5.5 / 55$ & $12 / 42$ \\
\hline $\begin{array}{l}\text { Horizontal limb-scans } \\
\text { separation }(\mathrm{km}) /(\mathrm{deg})\end{array}$ & $415 / 3.7$ & $320 / 2.9$ & $190 / 1.7$ \\
\hline
\end{tabular}

\footnotetext{
1 at tangent point.

2 see Fig. 1.
}

Irrespective of the specific retrieval algorithm, MIPAS observations are analyzed using a non-linear least squares fit based on the Gauss-Newton method (Ridolfi et al., 2000; Carlotti et al., 2006, 2007). The general iterative solution expression:

$$
\begin{aligned}
& \Delta \boldsymbol{x}=\left(\boldsymbol{x}_{i+1}-\boldsymbol{x}_{i}\right)=\left[\mathbf{K}^{\mathrm{T}} \mathbf{S}_{\mathrm{n}}^{-1} \mathbf{K}+\lambda \mathbf{I}+\mathbf{R}\right]^{-1} \\
& {\left[\mathbf{K}^{T} \mathbf{S}_{\mathrm{n}}^{-1} \boldsymbol{n}-\mathbf{R}\left(\boldsymbol{x}_{i}-\boldsymbol{x}_{a}\right)\right]}
\end{aligned}
$$

is used to compute, at iteration $i+1$, the correction $\Delta \boldsymbol{x}$ to the state vector $\boldsymbol{x}_{i}$. The state vector includes the values of the target quantities at all the geo-located retrieval grid points along the considered orbit. In Eq. (1) $\mathbf{K}$ is the Jacobian matrix containing the derivatives of the observations analyzed along the full orbit with respect to the elements of the state vector, calculated for $\boldsymbol{x}=\boldsymbol{x}_{i}, \mathbf{S}_{\mathrm{n}}$ is the variance-covariance matrix (VCM) of vector $\boldsymbol{n}$ that contains the differences between each observation and the corresponding simulation, I is the identity matrix, $\lambda$ is the Marquardt damping factor (Marquardt, 1963), $\mathbf{R}$ is an operator constraining the solution towards some selected feature (e.g. value) of an a-priori state $\boldsymbol{x}_{a}$. In the GMTR code the errors associated with the solution of the inversion procedure are characterized, at the last iteration, by the VCM of $\boldsymbol{x}$ :

$\mathbf{V}_{\Delta x}=\left[\mathbf{K}^{T} \mathbf{S}_{\mathrm{n}}^{-1} \mathbf{K}+\lambda \mathbf{I}+\mathbf{R}\right]^{-1}$

Matrix $\mathbf{V}_{\Delta x}$ maps the experimental random errors (represented by $\mathbf{S}_{\mathrm{n}}$ ) onto the uncertainty of the values of the retrieved parameters. In particular, the square root of the diagonal element of $\mathbf{V}_{\Delta x}$ provides the Estimated Standard Deviation (ESD or $\sigma$ ) of the corresponding parameter.

In operational retrievals the analysis is carried out on a limited number of narrow (less than $3 \mathrm{~cm}^{-1}$ wide) spectral intervals (Dudhia et al., 2002), called microwindows (MWs), that 
allow to limit the number of analyzed spectral elements and to avoid the analysis of spectral regions which are characterized by uncertain spectroscopic data, interference by nontarget species, or are influenced by unmodeled effects (Worden et al., 2004).

\subsection{Definition of spatial resolution}

In order to evaluate the vertical and horizontal resolution of the retrieval parameters, the averaging kernel matrix is computed by GMTR as:

$\mathbf{A}=\left[\mathbf{K}^{\mathrm{T}} \mathbf{S}_{\mathrm{n}}^{-1} \mathbf{K}+\lambda \mathbf{I}+\mathbf{R}\right]^{-1} \mathbf{K}^{T} \mathbf{S}_{\mathrm{n}}^{-1} \mathbf{K}$

In the 2-D analysis the retrieval is performed on a twodimensional grid in which each grid point $k$ is defined by its altitude $z_{\mathrm{k}}$ and its OC $\theta_{\mathrm{k}}$. The two-dimensional averaging kernel associated with parameter $k$ is the vector $\boldsymbol{a}(k, j)$, $j=1 \ldots p$ (where $p$ is the number of individual grid points) that corresponds to the $k^{\text {th }}$ row of matrix A. The vertical resolution of a retrieval parameter is defined as the FWHM of the bell-shaped feature identified by the subset of elements of vector $\boldsymbol{a}$ that correspond to the value $\theta_{\mathrm{k}}$ of the OC. In a similar way the horizontal resolution is defined as the FWHM of the subset of elements of $\boldsymbol{a}$ that correspond to the altitude $z_{\mathrm{k}}$.

\subsection{Information load}

In a 2-D approach it is possible to associate to each element of the atmospheric discretization a quantifier that measures the amount of information carried by that element with respect to a retrieval target (Carlotti and Magnani, 2009). The discretization of the atmosphere is operated on both the vertical and the horizontal domains (Carlotti et al., 2001). In the vertical domain altitude levels delimit layers as in a 1-D approach. The horizontal discretization is built using segments perpendicular to the Earth's geoid (radii) and extended up to the boundary of the atmosphere. The 2-D discretization leads to a web-like picture in which consecutive levels and radii define plane figures that are denoted as "cloves". It is then possible to assign to each clove $h$ the $\Omega$ quantifier defined as (Carlotti and Magnani, 2009):

$\Omega(q, h)=\left[\sum_{i=1}^{l} \sum_{j=1}^{m} \sum_{k=1}^{n}\left(\frac{\partial Y_{i j k}}{\partial q_{h}}\right)^{2}\right]^{1 / 2}$

where:

- $\Omega(q, h)=$ information load of clove $h$ with respect to atmospheric parameter $q$,

- $Y_{i j k}=$ spectral radiance of observation geometry $i$ at wavenumber $k$ of the analyzed MW $j$,

- $l=$ number of observation geometries that go through clove $h$,
- $m=$ number of analyzed MWs in observation geometry $i$,

- $n=$ number of spectral points in MW $j$.

In Eq. (4) quadratic summation has been chosen as combination rule of the partial derivatives because the column vector containing the set of elements within the triple summation is the Jacobian corresponding to the retrieval of target parameter $q$ in clove $h$. If we assume that the observations are uncorrelated and characterized by constant uncertainty $\left(\mathbf{S}_{\mathrm{n}}=\mathbf{I}\right)$ and we neglect external constraints, the term in square brackets of Eq. (2) turns into the scalar quantity (if only clove $h$ is considered):

$\left(\mathbf{K}^{\mathrm{T}} \mathbf{K}\right)_{h}=\sum_{i=1}^{l} \sum_{j=1}^{m} \sum_{k=1}^{n}\left(\frac{\partial Y_{i j k}}{\partial q_{h}}\right)^{2}$

(that this is only valid if the observations are uncorrelated, characterized by $\mathbf{S}_{\mathrm{n}}=\mathbf{I}$ and if constraints are neglected).

Therefore, in a retrieval analysis meant to determine the value of the target quantity $q$ in clove $h$ the uncertainty on the retrieved value would be given by $1 / \Omega$ (see Eq. 2). The value of $\Omega$ can be calculated for each clove of the 2-D discretization so that, for each retrieval target, we can draw a map of the 2-D distribution of the $\Omega$ quantifier (see Sect. 3.2 of Carlotti and Magnani, 2009). The $\Omega$ maps provide a picture of the "real" atmospheric sampling of the observations; they can be used to define optimal retrieval grids (where the information peaks) or to compare the atmospheric sampling relative to different targets or to different observation strategies. Therefore, the information load analysis is useful to predict the relative performance of the corresponding retrievals.

\section{Information-load analysis}

We have calculated 2-D maps of $\Omega$ for complete orbits operated with NOM, UTLS-1 and UTLS-2. Maps relative to all MIPAS main targets (see Sect. 3.1) have been compared in terms of intensity, altitude coverage and uniformity of the $\Omega$ distribution. Actually, in a conventional retrieval analysis, a better performance is expected for parameters located where $\Omega$ values are higher (see Sect. 5). For the calculation of $\Omega$ we used atmospheric fields corresponding to climatological profiles, taken from Remedios et al. (2007), relative to a January atmosphere. The MWs are those selected for the MIPAS operational retrievals. As an example, Fig. 2 shows the distribution of $\Omega$ with respect to the temperature along an orbit measured with the NOM observation mode. In Fig. 2 the vertical dimension of the atmosphere is expanded by a factor of 30 (for the sake of clarity) with respect to the extension of the Earth's radius. Values of the orbital coordinate are indicated in Fig. 2 for convenience. The $\Omega$ distributions have been calculated for a 2-D discretization of the atmosphere (Carlotti et al., 2001) operated with altitude levels evenly spaced by $1 \mathrm{~km}$ and radii evenly spaced by 0.25 latitudinal degrees 


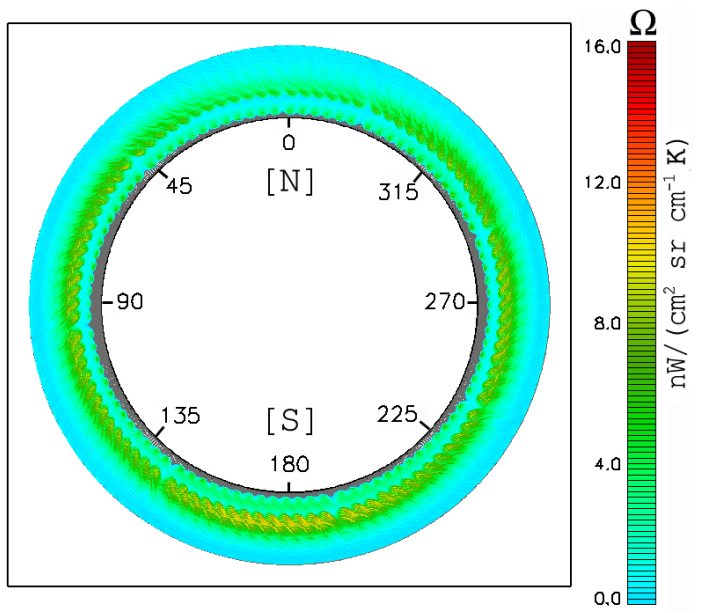

Fig. 2. Distribution of $\Omega$ with respect to the temperature along a MIPAS orbit measured with the NOM observation mode. Values of the $\mathrm{OC}$ and position of the geographical poles are reported within the figure. The vertical extension of the atmosphere (from the surface up to $80 \mathrm{~km}$ ) is expanded by a factor of 30 with respect to the extension of the Earth's radius.

(about $28 \mathrm{~km}$ ). A comparison of $\Omega$ for temperature in the three observation modes is shown in the panels of Fig. 3 that reports (with equal color scales) a zoom-in of the $\Omega$ distributions around the South Pole in the case of NOM (left-), UTLS-1 (middle-) and UTLS-2 (right-panel); in this figure the vertical dimension of the atmosphere is expanded by a factor of 10. In order to evaluate the altitude extension of $\Omega$, in Fig. 3 (as well as in the following Fig. 4) altitude levels from 10 to $50 \mathrm{~km}$ at $5 \mathrm{~km}$ steps are indicated. It can be seen in Fig. 3 that the three observation modes differ in intensity, altitude extension and uniformity of the $\Omega$ distribution. In general, in the UT/LS, the UTLS- $2 \Omega$ map shows higher intensities and better uniformity. The latter property allows to select the retrieval grid without taking care to match the positions where the information accumulates (Carlotti and Magnani, 2009). In these cases the choice of the retrieval grid can be operated on the basis of only the stability of the retrieval as determined by the trade-off between precision and spatial resolution (Carlotti et al., 2007).

The visual inspection of maps as those in Figs. 2 and 3 gives a first indication about the relative performance expected in the retrievals. A more definite indication can be obtained looking at the difference between the $\Omega$ distributions that are generated by two different observation modes for a specific target. A first example of comparison is given in the left panel of Fig. 4 that shows the difference between the $\mathrm{HNO}_{3} \Omega$ distributions generated by UTLS- 1 and NOM. In order to facilitate the grasp, only positive values are represented in the map. In Fig. 4 the top of the color scale is about $15 \%$ of the maximum $\mathrm{HNO}_{3} \Omega$ value generated by UTLS1. The distribution of positive differences between 10 and $45 \mathrm{~km}$ suggests, in this altitude interval, a better quality of the $\mathrm{HNO}_{3}$ VMRs retrieved from UTLS-1 observations with respect to those retrieved from NOM observations. Positive differences between UTLS- 1 and NOM $\Omega$ distributions are widespread, up to $50 \mathrm{~km}$, also for the other targets even if with different intensities and uniformities. As a second example, the right panel of Fig. 4 reports, with the same format as the left panel, the difference between the $\Omega$ distributions generated by UTLS- 2 and those generated by UTLS- 1 in the case of $\mathrm{O}_{3}$. In this panel the top of the color scale is about $22 \%$ of the maximum $\mathrm{O}_{3} \Omega$ value generated by UTLS- 2 . Positive values are clearly evident in the right panel of Fig. 4 between 15 and $45 \mathrm{~km}$. Also for the other targets the $\Omega$ differences inspection indicates that, in the lower stratosphere, the $\Omega$ distribution for UTLS- 2 observations is comparable and sometimes better than that for the UTLS-1 observations. The complementary maps (not shown) reporting only the negative values of the differences confirm the statements above.

The information-load analysis on the eight MIPAS main targets indicates that:

1. the performance of the two UT/LS modes is competitive with that of the NOM mode even in altitude ranges that exceed the UT/LS region,

2. in the UT/LS some of the UTLS-2 products should be of better quality than the corresponding UTLS-1 products.

\section{Simulated retrievals}

The results of the information-load analysis can be verified by investigating retrievals performed for simulated observations. The steps of this kind of retrieval are:

1. generate simulated observations with the GMTR standalone forward model; reference altitude profiles are used for the target quantity in this step. The observational parameters are taken from a real MIPAS orbit,

2. perform the retrieval analysis on the simulated observations using an initial guess obtained by applying random perturbations to the reference profiles,

3. evaluate the retrieval precision by comparing the retrieved values with the reference values used to generate the simulated observations,

4. evaluate the horizontal and the vertical resolution of the retrieval products by means of the 2 -D averaging kernels.

As for the information-load analysis we have used the operational MWs and the climatological profiles taken from Remedios et al. (2007). This atmospheric model includes six latitudinal bands (without day/night discrimination) whose latitudinal extents range from 20 to $35 \mathrm{deg}$. Since the largest separation in our retrieval grids (see following sections) is 

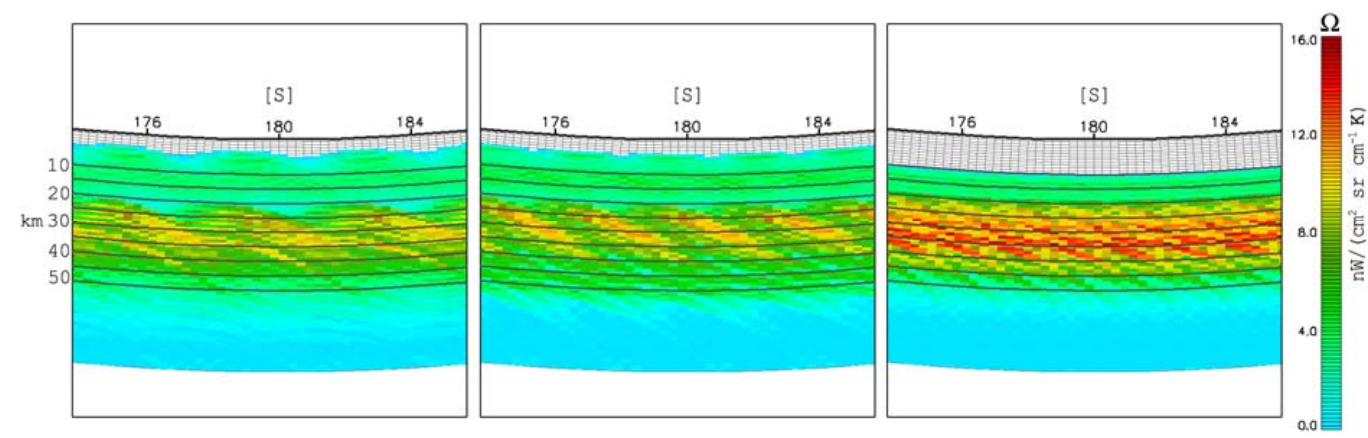

Fig. 3. Zoom-in of the $\Omega$ distributions with respect to temperature around the South Pole in the case of NOM (left-), UTLS-1 (middle-) and UTLS-2 (right-panel). The vertical dimension of the atmosphere is expanded by a factor of 10 with respect to the extension of the Earth's radius.
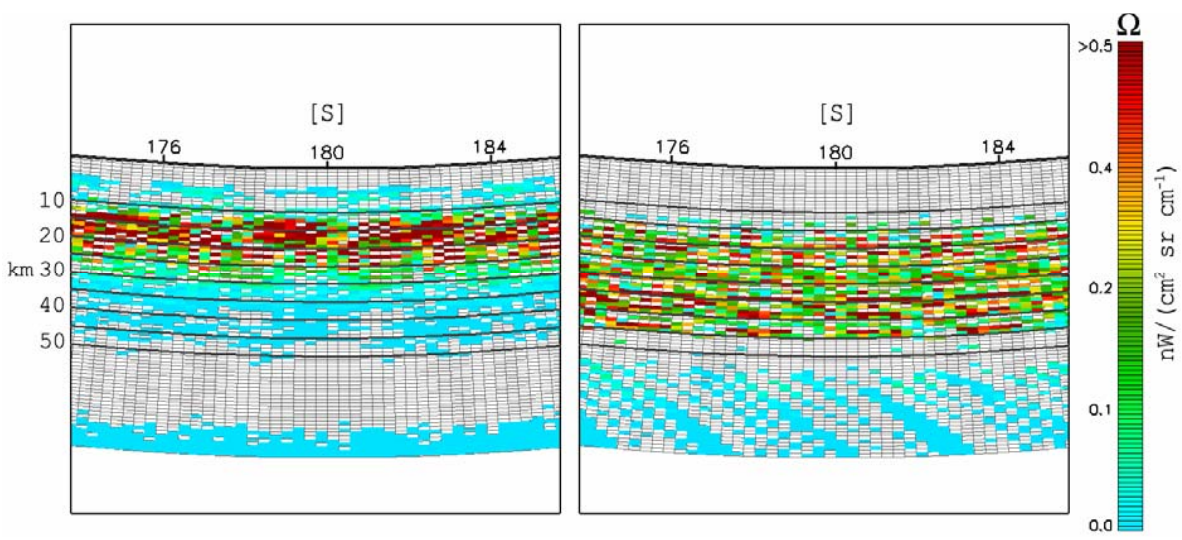

Fig. 4. Left panel: difference between the $\Omega$ distributions generated by UTLS-1 and NOM observation modes for the $\mathrm{HNO}_{3}$ VMR. Right panel: difference between the $\Omega$ distributions generated by UTLS- 2 and UTLS- 1 in the case of $\mathrm{O}_{3}$. The vertical dimension of the atmosphere is expanded by a factor of 10 with respect to the extension of the Earth's radius.

less than 3.8 deg the contribution of smoothing errors is negligible in our simulated retrievals. For all the tests reported in this section the maximum allowed perturbations applied in step 2 were $5 \%, 1 \%$, and $50 \%$ for pressure, temperature, and VMR profiles, respectively. Maximum perturbations of $80 \%$ were applied to atmospheric continuum profiles which, to simulate real retrievals, are also included in the state vector $\boldsymbol{x}_{i}$ of Eq. (1). Larger perturbations induce an increase in the number of retrieval iterations and lead to results that differ within their estimated standard deviation.

\subsection{Performance on the NOM retrieval grid}

In order to verify whether the two UT/LS modes are competitive with NOM at altitudes that exceed the UT/LS region we have evaluated the performance of the three modes in the retrieval conditions that are operationally used for the NOM. A common retrieval grid has been used with altitude steps defined by the geometrical separation between the NOM observation geometries (with the exception for the lower limit of $12 \mathrm{~km}$ adopted for UTLS-2 due to its limb-scanning pattern (see Fig. 1)); in the horizontal domain 96 profiles were retrieved at the NOM natural grid (see Sects. 2 and 3.1). Common MWs and auxiliary data (those adopted for the NOM operational analyses) have been used in the simulated retrievals reported in this and in the next sub-section. No constraints to the solution were imposed for the main targets in all the simulated retrievals presented in this paper $(\mathbf{R}=\mathbf{0}$ in Eq. (1)). Weak a-priori information was adopted in order to stabilize the retrieval of atmospheric continuum parameters (using $\lambda=0.1$ as a damping factor and model profiles from Clough et al. (1989) weighted with $200 \%$ uncertainty).

An example of the results of a simulated retrieval is given in Fig. 5 which refers to $\mathrm{N}_{2} \mathrm{O}$ VMR retrieved from UTLS-2 observations (since the behaviour of $\mathrm{N}_{2} \mathrm{O}$ is quite representative we will use this target also for the following examples). Panel (a) of Fig. 5 shows the retrieved VMRs, panel (b) the absolute value of the difference between retrieved and reference VMRs, panels (c) and (d) the vertical and the horizontal resolution of the retrieval products, respectively. In panel (d) of Fig. 5 are present vertical stripes that correspond to the wider separations between the limb-scans (see Sect. 2). 

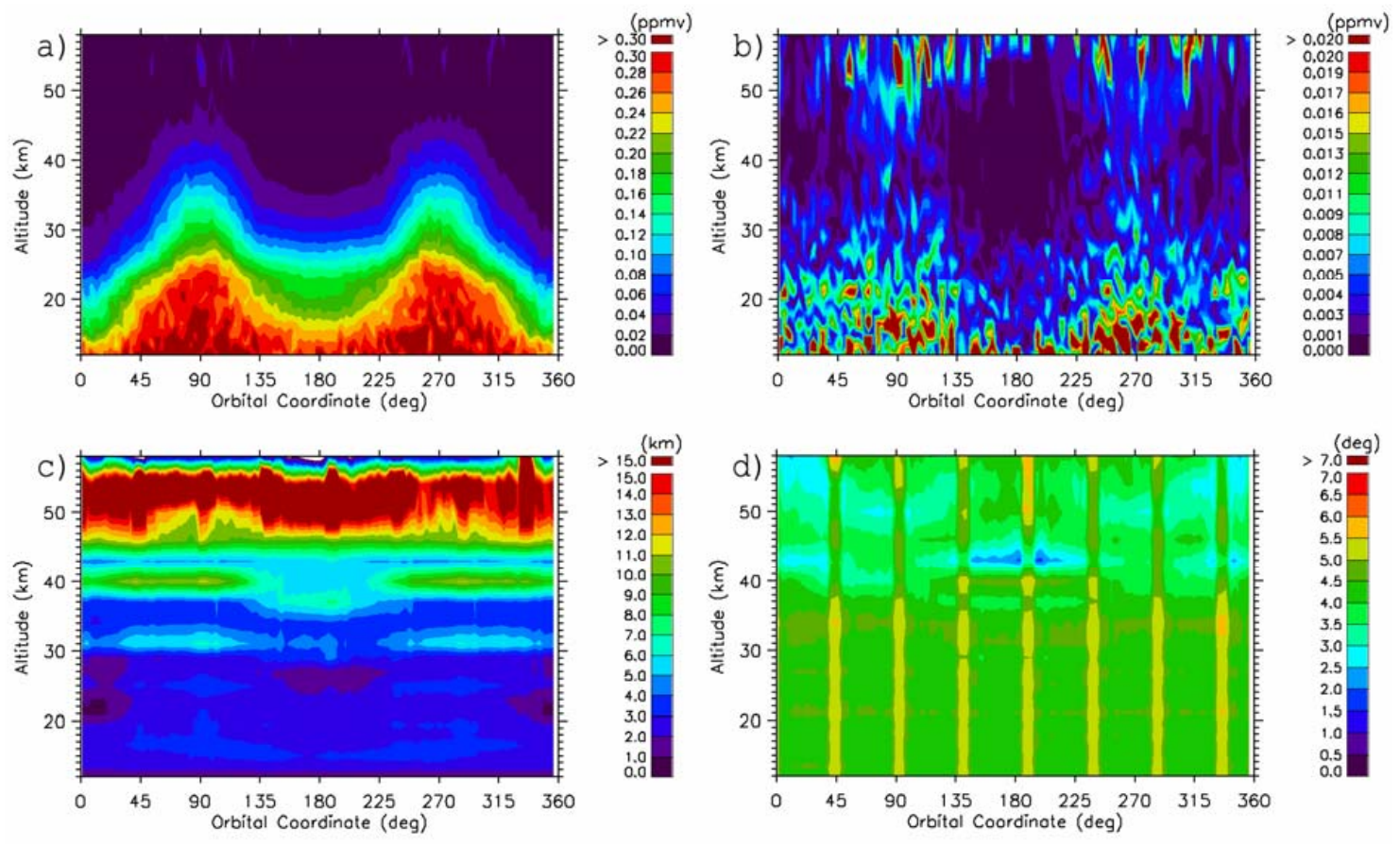

Fig. 5. Results relative to the retrieval of $\mathrm{N}_{2} \mathrm{O}$ VMR from UTLS-2 observations. Panel (a) retrieved values, panel (b) absolute value of the difference between retrieved and reference VMRs, panel (c) vertical resolution, panel (d) horizontal resolution.

Figure 5 shows that $\mathrm{N}_{2} \mathrm{O}$ can be retrieved from UTLS-2 with acceptable precision well above the highest tangent altitude of the observations $(\sim 42 \mathrm{~km})$. Above this altitude the vertical resolution worsens (as expected) but seems to improve again at the topmost layer. The horizontal resolution, in turn, shows improvement just above the highest tangent altitude. These unexpected behaviors can be explained by considering that at higher altitudes the information load decreases due to the decreasing $\mathrm{N}_{2} \mathrm{O}$ VMR (see panel (a)); in these conditions the subset of elements of vector $\boldsymbol{a}$ of the averaging kernel depicts a distorted feature that is no longer bell-shaped as required by the definition of spatial resolution given in Sect. 3.2. Such a situation is generally encountered where the information load is weak and/or irregularly scattered.

In order to provide an overall picture of the performance of all the main targets (with the exception of pressure), the panels of Fig. 6 show the standard deviation of the difference between retrieved and reference profiles (left column), the average horizontal resolution (middle column) and the average vertical resolution (right column) for NOM (red-), UTLS-1 (green-) and UTLS-2 (blue-lines). In Fig. 6 the spatial resolution is shown only at altitudes where the quantifier is meaningful (see previous paragraph). We notice that below $10 \mathrm{~km}$ the precision of the retrievals (panels in the left column) relative to NOM and UTLS-1 have reduced validity due to the waving shape of the tangent altitudes along the orbit (see upper- and middle-panels of Fig. 1) that reduces the statistics at those altitudes. Figure 6 shows that:
- the precision of UTLS-1 (in terms of standard deviation of the difference between retrieved and reference profiles) is comparable to that of NOM in the full altitude range for all the targets but $\mathrm{HNO}_{3}$ for which UTLS1 shows a better precision. The precision of UTLS-2 is comparable to that of NOM up to altitudes of about $45 \mathrm{~km}$,

- the horizontal resolution of the UTLS-1 products is generally better than that of the NOM products in the full altitude range. The horizontal resolution of the two $\mathrm{UT} / \mathrm{LS}$ modes is comparable in the altitude range where the UTLS-2 quantifier is meaningful,

- the vertical resolution of the three observation modes is comparable up to about $30 \mathrm{~km}$; above this altitude NOM provides a better performance than the other two for all the targets but $\mathrm{HNO}_{3}$ for which NOM and UTLS-1 are comparable.

In general we verify that UTLS-1 is competitive with NOM in the full altitude range despite the reduced altitude extension and the larger steps of its limb-scanning pattern above $30 \mathrm{~km}$ (see Sect. 2). In the particular case of $\mathrm{HNO}_{3}$ the UTLS-1 precision is clearly better than that of NOM in the entire retrieval range while both spatial resolutions are comparable. This outcome confirms the indication provided by the information-load analysis for this target. Regarding the comparison between the performance of UTLS-2 and NOM, 

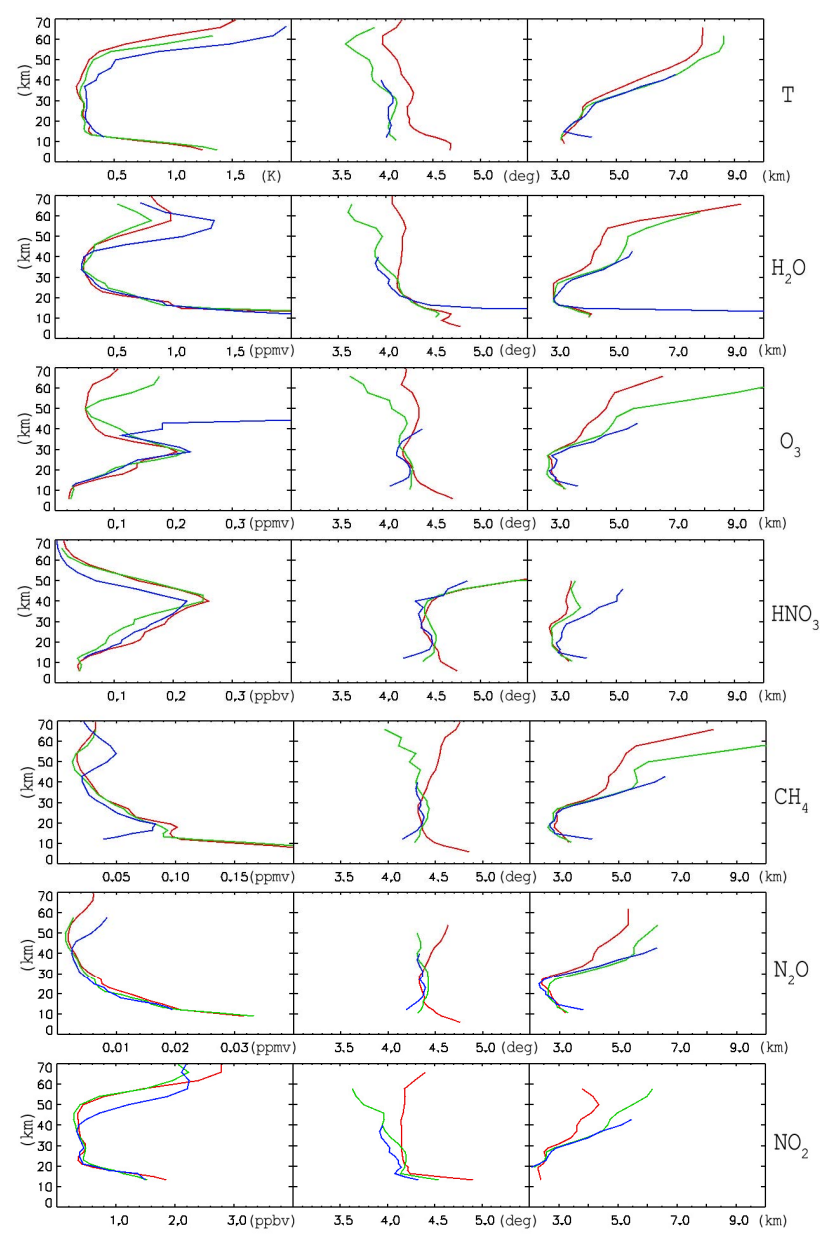

Fig. 6. Standard deviation of the difference between retrieved and reference profiles (left column), average horizontal resolution (middle column) and average vertical resolution (right column) for NOM (red), UTLS-1 (green) and UTLS-2 (blue). Target is identified on the right side.

considerations similar to those reported for UTLS-1 apply at altitudes above $10 \mathrm{~km}$ (lower limit of UTLS-2 observations) and below about $40 \mathrm{~km}$ (where the spatial resolution of this observation mode cannot be evaluated).

The overall results of the above comparison performed on the NOM retrieval grid support the reliability of the information-load analysis that indicates (see Sect. 3) a competitive performance of the two UT/LS observation modes at altitudes that exceed the UT/LS region.

\subsection{Performance in the UT/LS}

Focusing on the UT/LS region (below $25 \mathrm{~km}$ ) we first investigate the performance of each observation mode when its natural grid (see Sect. 3.1) is used in the horizontal domain for the retrievals. In the vertical domain the profiles are defined at the same altitudes as in Sect. 5.1 up to $25 \mathrm{~km}$ and, above, at the tangent altitudes of the specific observation mode. The natural grids have (in accordance with the observational parameters given in Sect. 2): 96 profiles separated by about $415 \mathrm{~km}$ for NOM, 125 profiles separated by about $320 \mathrm{~km}$ for UTLS-1, and 213 profiles separated by about $190 \mathrm{~km}$ for UTLS-2 retrievals.

In order to asses the appropriateness of the natural retrieval grids, we have investigated the behaviour of the horizontal resolution of the retrieval products obtained on these grids. The left panel of Fig. 7 shows, for an OC interval of $50 \mathrm{deg}$, the horizontal resolution of $\mathrm{N}_{2} \mathrm{O}$ VMRs when retrieved from UTLS-2 observations using its natural retrieval grid. The colour alternation that appears in this map along the OC indicates that the resolution requirements of the UTLS-2 natural grid trigger a retrieval instability with this number of retrieval parameters. The observed oscillation can be reduced by increasing the horizontal separation of the profiles (Carlotti et al., 2007) (we have observed in Sect. 4 that in the case of UTLS-2 the retrieval grid can be defined arbitrary because of the uniform $\Omega$ distributions that it generates). Following this strategy we have found that a separation of $2.25 \mathrm{deg}$ (160 profiles separated by about $250 \mathrm{~km}$ ) leads to a satisfactory stability of the UTLS-2 retrievals. An example of the improvement is given in the right panel of Fig. 7 that shows, at $18 \mathrm{~km}$, the horizontal resolution extracted from the map in the left panel (green line) and the horizontal resolution when the 2.25 deg separation is used for the horizontal grid (red line).

As for the performance of UTLS-1 Fig. 8 shows, in its left panel, the horizontal resolution of $\mathrm{N}_{2} \mathrm{O}$ VMRs when retrieved from UTLS-1 observations using their natural grid. It can be seen in Fig. 8 that the retrieval is rather stable indicating that the geometrical separations of the UTLS-1 natural grid are consistent with the information load of the observations.

In a further step we have explored the possibility to improve the horizontal resolution of the UTLS-1 products by reducing the geometrical steps in the horizontal retrieval grid. We have found that even moderate reductions of the horizontal step trigger instabilities that, as in the UTLS-2 case, appear as oscillations. As an example we show in the right hand panel of Fig. 8 the values of the horizontal resolution at $18 \mathrm{~km}$ extracted from the map in the left hand panel (green line) and the values, at the same altitude, when the $2.25 \mathrm{deg}$ separation is used for the horizontal grid (red line).

The behaviour of the vertical resolution reflects the one of 1-D retrievals when the horizontal grid does not trigger the instabilities discussed in the previous paragraph. Oscillations appear also in the vertical domain when the horizontal resolution becomes markedly unstable.

The analysis that we have shown for $\mathrm{N}_{2} \mathrm{O}$ is basically representative of the behaviour of the other main MIPAS targets with the exception of $\mathrm{NO}_{2}$ that, in the case of UTLS-2 suffers from instabilities in the vertical domain. To sum up, we can state that in the UT/LS the natural grid is suitable for the retrievals on UTLS-1 observations while the $2.25 \mathrm{deg}$ grid 

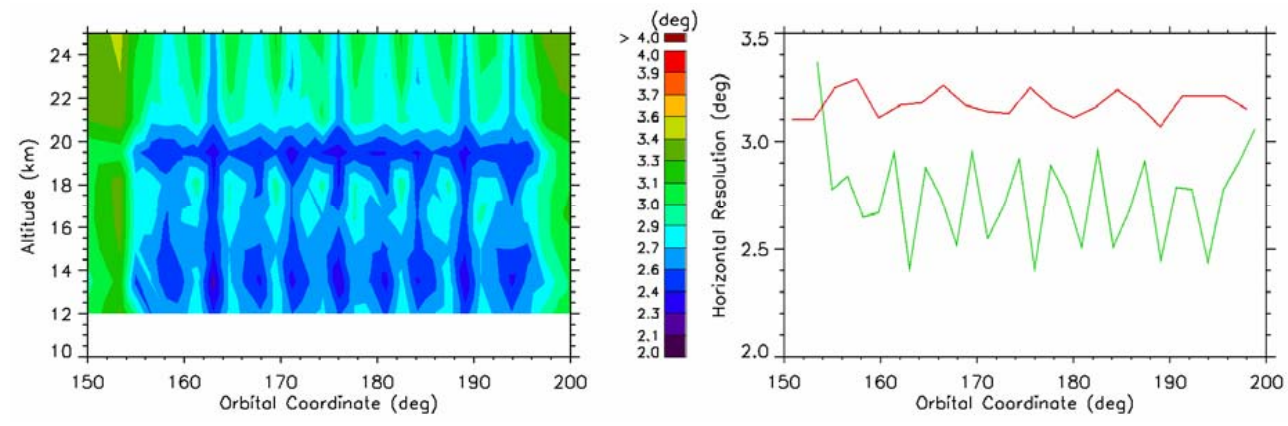

Fig. 7. Left panel: horizontal resolution of $\mathrm{N}_{2} \mathrm{O}$ VMRs when retrieved from UTLS-2 observations using the natural retrieval grid. Right panel: values of the horizontal resolution at $18 \mathrm{~km}$ extracted from the map in the left panel (green line) and values, at the same altitude, when the 2.25 deg separation is used for the horizontal grid (red line).
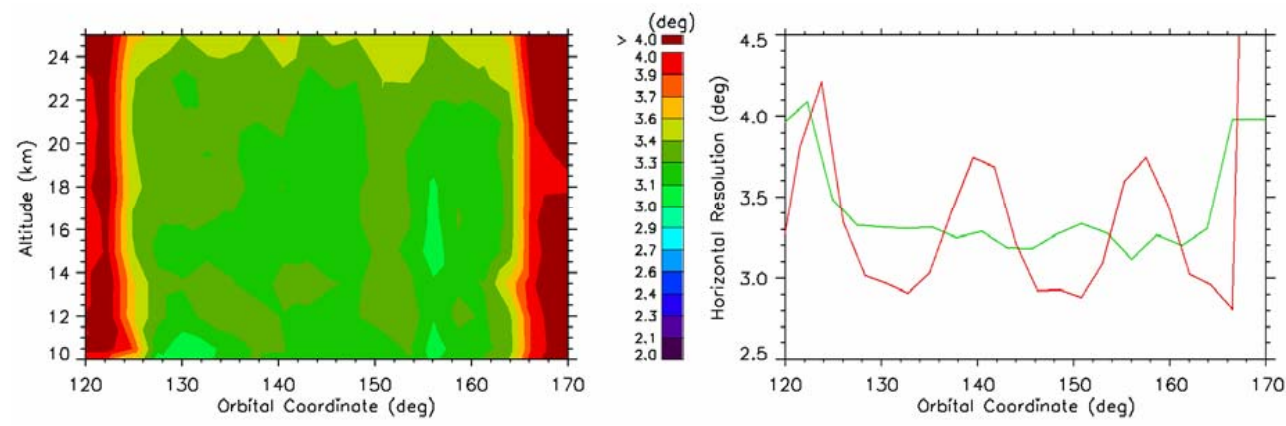

Fig. 8. Left panel: horizontal resolution of $\mathrm{N}_{2} \mathrm{O}$ VMRs when retrieved from UTLS-1 observations using the natural retrieval grid. Right panel: values of the horizontal resolution at $18 \mathrm{~km}$ extracted from the map in the left panel (green line) and values, at the same altitude, when the $2.25 \mathrm{deg}$ separation is used for the horizontal grid (red line).

provides a satisfactory trade-off in the case of UTLS-2 observations. The performance obtained using these grids is shown in Fig. 9 that reports the same quantifiers (and with the same formats) as in Fig. 6. In Fig. 9 the plots for NOM are also included for reference; these plots are just a zoom-in of the plots in Fig. 6 since they refer to retrievals carried out using (as in Fig. 6) the natural grid of the NOM observation mode. The analysis of Fig. 9 shows that in the UT/LS:

- UTLS-1 can provide, for all targets, 125 profiles with a precision which is comparable to the one of the 96 profiles of NOM. The UTLS-1 profiles are characterized by horizontal resolution which is about $30 \%$ better than that of NOM. The UTLS-1 vertical resolution is generally better than that of NOM in all the altitude range,

- UTLS-2 can provide 160 profiles that do not extend below $12 \mathrm{~km}$. For $\mathrm{H}_{2} \mathrm{O}, \mathrm{O}_{3}$ and $\mathrm{NO}_{2}$, the UTLS-2 precision is comparable to that of UTLS- 1 . The UTLS-2 precision is worse than that of UTLS- 1 by about $45 \%$ for $\mathrm{HNO}_{3}$ and $25 \%$ for the other targets. For all targets the UTLS-2 horizontal resolution is better than that of UTLS-1 especially (with the exception of $\mathrm{H}_{2} \mathrm{O}$ ) at low altitudes. The vertical resolution of UTLS-2 is compa- rable to that of UTLS-1 up to about $20 \mathrm{~km}$; it is worse above.

In general we show in the simulation studies that, as also indicated by the information-load analysis, UTLS-2 gives an overall performance which is competitive with that of UTLS1. In particular UTLS-2 products are preferable when the horizontal resolution is a requirement. The competitive quality of the UTLS-2 products is obtained despite the reduced altitude extension and the larger altitude steps of the limbscanning pattern of this observation mode (see Sect. 2).

\section{Conclusions}

We have assessed the performance of the three MIPAS observation modes that sound the atmosphere in the UT/LS region when their observations are analyzed with a 2-D tomographic algorithm. For the purpose we first carried out the information-load analysis for all the MIPAS main targets using the observational parameters of real orbits operated with the three observation modes. The information-load analysis indicates that: 

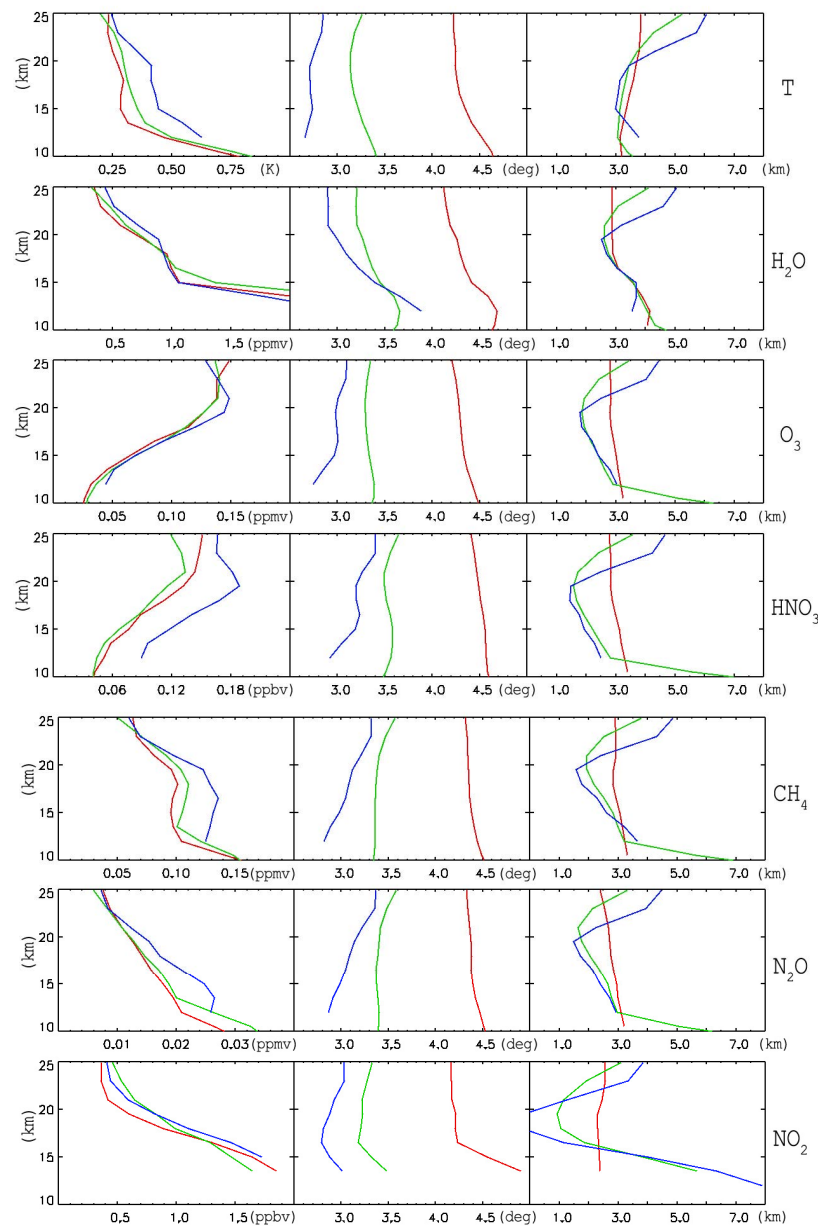

Fig. 9. Standard deviation of the difference between retrieved and reference profiles (left column), average horizontal resolution (middle column) and average vertical resolution (right column) for NOM (red), UTLS-1 (green) and UTLS-2 (blue). NOM and UTLS-1 plots refer to their natural retrieval grids, UTLS-2 plots refer to the 2.25 deg retrieval grid.

- the performance of the two UT/LS modes is competitive with that of the NOM mode even in altitude ranges that exceed the UT/LS region,

- in the UT/LS the information-load distributions generated by UTLS- 2 show, in general, higher intensities and better uniformity than those generated by UTLS -1 . The high uniformity indicates that for the UTLS-2 analyses the retrieval grid can be selected on the basis of only the trade-off between precision and spatial resolution.

Second, in order to validate the qualitative outcomes of the information-load analysis and to get quantitative estimates about the performance of the three observation modes, we have carried out simulated retrievals. In simulation studies we have evaluated precision and spatial resolutions of the MIPAS targets retrieved from each observation mode.
The results obtained with the simulated retrievals confirm the indications of the information-load analysis. In particular they have shown that:

- the quality of the profiles retrieved from the two observation modes that were specifically designed for the UT/LS region is similar to that retrieved from the NOM mode (that was designed for the whole stratosphere) up to altitudes that far exceed the UT/LS,

- in the UT/LS the performance of the two specific observation modes is comparable. In terms of horizontal resolution the best performance is provided by the UTLS-2 mode that was excluded by ESA from the current MIPAS duty cycle.

These results have been obtained with the analysis of the MWs used for the MIPAS operational retrievals. Different MWs could be selected in order to enhance the performance of a specific observation mode in the altitude range of interest. In this task of selection the performance of different sets of MWs can be tested with the information-load analysis that, as we have shown in this study, has proven to be a reliable tool for a qualitative assessment of the retrieval potential and for the selection of the retrieval grid.

Acknowledgements. E. Papandrea acknowledges support by ESA within the framework of the Changing Earth Science Network Initiative.

Edited by: C. von Savigny

\section{References}

Carlotti, M.: Global-Fit Approach to the Analysis of Limb-scanning Atmospheric Measurements, Appl. Opt., 27, 3250-3254, 1988.

Carlotti, M., Dinelli, B. M., Raspollini, P., and Ridolfi, M.: Geofit Approach to the analysis of limb-scanning satellite measurements, Appl. Opt., 40, 1872-1885, 2001.

Carlotti, M., Brizzi, G., Papandrea, E., Prevedelli, M., Ridolfi, M., Dinelli, B. M., and Magnani, L.: GMTR: two-dimensional multitarget retrieval model for MIPAS/ENVISAT observations, Appl. Opt., 45, 716-727, 2006.

Carlotti, M., Dinelli, B. M., Papandrea, E., and Ridolfi, M.: Assessment of the horizontal resolution of retrieval products derived from MIPAS observations, Opt. Express, 15, 10458-10472, 2007.

Carlotti, M. and Magnani, L.: Two-dimensional sensitivity analysis of MIPAS observations, Opt. Express, 17, No. 7, 5340-5357, 2009.

Clough, S. A., Kneizys, F. X., and Davis, R. W.: Line shape and the water vapor continuum, Atmos. Res., 23, 229-241, 1989.

Dudhia, A., Jay, V. L., and Rodgers, C. D.: Microwindow selection for high-spectral-resolution sounders, Appl. Opt., 41, 36653673, 2002.

Fischer, H., Birk, M., Blom, C., Carli, B., Carlotti, M., von Clarmann, T., Delbouille, L., Dudhia, A., Ehhalt, D., Endemann, M., Flaud, J. M., Gessner, R., Kleinert, A., Koopman, R., Langen, 
J., López-Puertas, M., Mosner, P., Nett, H., Oelhaf, H., Perron, G., Remedios, J. J., Ridolfi, M., Stiller, G., and Zander, R.: MIPAS: an instrument for atmospheric and climate research, Atmos. Chem. Phys., 8, 2151-2188, doi:10.5194/acp-8-2151-2008, 2008.

Marquardt, D. W.: An algorithm for the least-squares estimation of non-linear parameters, SIAM J. Soc. Appl. Math., 11, 431-441, doi:10.1137/0111030, 1963.

Remedios, J. J., Leigh, R. J., Waterfall, A. M., Moore, D. P., Sembhi, H., Parkes, I., Greenhough, J., Chipperfield, M. P., and Hauglustaine, D.: MIPAS reference atmospheres and comparisons to V4.61/V4.62 MIPAS level 2 geophysical data sets, Atmos. Chem. Phys. Discuss., 7, 9973-10017, doi:10.5194/acpd7-9973-2007, 2007.
Ridolfi, M., Carli, B., Carlotti, M., von Clarmann, T., Dinelli, B. M., Dudhia, A., Flaud, J. M., Hoepfner, M., Morris, P. E., Raspollini, P., Stiller, G., and Wells, R. J.: Optimized forward model and retrieval scheme for MIPAS near-real-time data processing, Appl. Opt., 39, 1323-1340, 2000.

Rodgers, C. D.: Inverse methods for atmospheric sounding: Theory and Practice, Series on Atmospheric, Oceanic and Planetary Physics, Vol. 2, World Scientific Publishing Co., Singapore, 65$100,2000$.

Worden, J. R., Bowman, K. W., and Jones, D. B.: Two-dimensional characterization of atmospheric profile retrievals from limb sounding observations, J. Quant. Spectrosc. Radiat. Transfer, 86, 45-71, 2004. 